\title{
FEATURED EDITORIAL
}

\section{The greying of cardiology: implications for management}

\author{
Nanette K Wenger
}

"The development of new therapies and devices should reflect the dominant population that will ultimately use them - in the case of cardiovascular therapies and devices, this is exemplified by elderly patients"

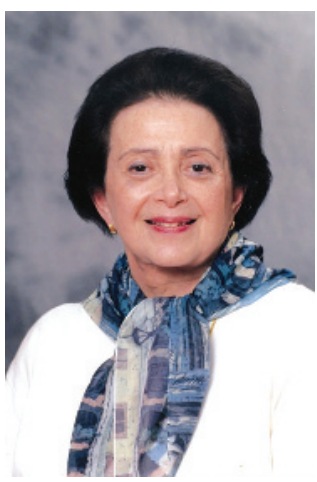

C ardiovascular disease, as well as constituting the largest health burden at elderly age, is currently a disease encountered primarily in the elderly population. Almost half of all octogenarians have clinical manifestations of coronary heart disease, cerebrovascular disease, peripheral arterial disease, and/or heart failure. Hypertension is present in more than half of the population older than age 65 , and atrial fibrillation is noted in about $10 \%$ of those aged 80 years and older. Thus it is not surprising that elderly patients populate our coronary and intensive care units, our operating suites, our diagnostic laboratories and our emergency departments. The octogenarians who comprise $5 \%$ of the US population account for more than $20 \%$ of all hospital admissions for myocardial infarction and at least one-third of all infarctionrelated hospital deaths. Although heart failure is present in about $10 \%$ of persons older than age 75 years, this elderly subset accounts for $50 \%$ of all heart failure hospitalisations.

\section{INCREASING BURDEN OF HEART VALVE DISEASE}

Notwithstanding that coronary heart disease is the major cardiovascular problem, a recent publication $^{1}$ addresses the increasing burden of heart valve disease with aging. The authors suggest that more than one in eight people aged 75 years and older have moderate or severe heart valve disease and constitute another target for research studies. There is twice as much heart valve surgery in the US Medicare population older than age 65 compared with that at ages $45-64$ years. ${ }^{2}$

Much of the contemporary increase in longevity is related to the effective prevention and treatment of cardiovascular disease, and in particular coronary heart disease at younger ages; nonetheless cardiovascular disease remains the major cause of death, disability and healthcare use for elderly patients. Effective coronary preventive interventions among aged persons have the potential to show greatest benefit, as the attributable risk for any risk factor is greater at elderly age. The American Heart Association has emphasised secondary prevention for patients 75 years of age or older, ${ }^{3}$ but data are limited regarding applicability to the advanced elderly. Are there markers or subclinical characteristics that can focus the
Heart 2007:93:411-412. doi: 10.1136/hrt.2006.113852

selection both of preventive interventions and of other therapies for very elderly patients?

The paucity of data to guide clinical cardiovascular management puts elderly patients at a disadvantage worldwide. Is the current accelerated application of high technology diagnostic and therapeutic procedures appropriate for the elderly and very elderly, and how can we identify those patients most likely to benefit? Increasingly, outcomes for patients aged $65-75$ years correspond more closely to those of their younger counterparts, but the information necessary to trigger changes in the care of the advanced elderly patients is distressingly lacking.

\section{GROWING POPULATION OF ELDERLY}

The ageing of the population globally, but in particular among industrialised nations, and the explosion in the absolute numbers of elderly persons is a societal phenomenon that has no historical precedent. The contemporary life expectancy of an 80-year-old man in the USA is about 7 years and that of an 80-year-old woman is 9.1 years. This evolving and greying landscape has challenged physicians and researchers to provide a science-centred, patient-focused pattern of care for the oldest old. A pivotal problem is the lack of adequate information about cardiovascular disease in aged patients necessary to respond to this demographic. Given that the clinical presentations, the disease severity, the clinical course, the gender distribution and the prognosis of cardiovascular illnesses vary substantially at elderly compared with younger age, how can guidelines be developed to inform clinical practice as to appropriate indications for testing and therapy and subsequently as to the optimal choice among testing and therapeutic modalities? For the same cardiovascular disease entity, the presentation at elderly age must incorporate its superimposition on the agerelated changes in the cardiovascular system; the diminished multi-system organ reserve; and the polypharmacy characteristic among elderly patients; as well as combined cardiovascular comorbidities. With the exponential escalation of interventional and high technology procedures in the US Medicare population, how can we best define the differential benefit or risk of each at advanced age? How can we best evaluate the candidacy of an individual elderly patient for a specific diagnostic or therapeutic procedure?

A scientific database is urgently needed to shape medical and societal decisions regarding healthcare for elderly patients-both preventive cardiovascular care and use of specific cardiovascular therapies. We must increase the scientific knowledge about the impact of cardiovascular medical 
and surgical strategies on morbidity, on functional status and on quality of life-in contrast to the (albeit meager) mortality data currently available. This must be done in the context of incorporating the health values, health beliefs and desired outcomes of individual elderly patients. A major challenge is the dearth of information about the preferences and health values of the oldest old, paramount to guide their healthcare in general and their cardiovascular care in particular. Elderly patients are a highly heterogeneous group regarding their severity of illness; their functional and cognitive status; their psychosocial needs; and their likely expectations of medical care. What risks are elderly patients (or is an individual elderly patient) willing to incur for symptom amelioration, for maintenance of health? Alternatively, what levels of symptoms or illness are tolerable for those who are risk averse?

ENROL THE ELDERLY IN CLINICAL RESEARCH STUDIES The demographic imperative to include elderly patients in contemporary clinical trials of cardiovascular therapies is that they represent the majority of the population likely to receive such treatments. ${ }^{2}$ Respect and justice for aged patients, and social and societal responsibility for their care, are laudatory abstract concepts, but implementation is likely to fail unless it is data driven. The challenge is the enrolment and maintenance of elderly patients with cardiovascular illness in clinical research studies that will provide guidance as to which preventive, diagnostic and therapeutic cardiovascular interventions can prolong meaningful life; can lessen disability and dependency; can decrease costly hospitalisations; and can improve functional capacity and personal satisfaction. ${ }^{4}$ Improvement of functional independence and life quality may be more valued than marginal longevity benefit.

The results of clinical trials can improve clinical practice by guiding clinical decision-making. Possibly the lesser use of proved beneficial cardiovascular therapies in elderly patients, and in particular in the highest risk elderly patients, reflects their exclusion from or under-representation in the clinical research studies that documented such benefit. Efforts must be mounted to enhance the recruitment and enrolment of elderly patients so that favourable trial results are unequivocally generalisable. As an example, in the CRUSADE quality improvement initiative ${ }^{5}$ a quarter of the patients with an acute coronary syndrome were older than age 70 and 5\% were older than age 90 years. The use of lipid lowering drugs, known to decrease mortality in acute coronary syndromes, was $70 \%$ in a younger population as compared with $53 \%$ at age older than 75 years.

Categories of research answers that might determine comparable cardiovascular care at an elderly age to that at younger age include those that address the effectiveness of preventive interventions, the appropriate use of diagnostic testing, the identification of high risk asymptomatic or minimally symptomatic elderly individuals, and the relative risks and benefits of drug regimens and interventional procedures. The development of new therapies and devices should reflect the dominant population that will ultimately use them-this is exemplified by elderly patients in the case of cardiovascular therapies and devices, yet elderly patients remain under-represented in these research studies.

\section{THE CHALLENGE OF INNOVATION}

Governments and social institutions comparably face the challenge of innovation as healthcare needs and costs at elderly age escalate, but lack the knowledge and the adequate experience about the social changes appropriate to address the burgeoning health needs of the oldest old. Neither can business, industry or the medical community provide evidenceor experience-based guidance. How can we appropriately reform public and private healthcare institutions and policies to meet the cardiovascular care needs of this landslide population?

In contrast to the Ponce de Leon quest for the fountain of youth, a provocative contemporary quest in the realm of the unknown would be for interventions that can enhance successful aging, lifestyle and environmental changes that may protect and preserve health into elderly age.

Research grants/contracts/trial, Steering Committee: Eli Lilly, Astra Zeneca, Pfizer

Consultantship: Cardiology Consultant, Bristol Myers Squibb; Women's Advisory Board, CV Therapeutics; Sanofi-Aventis; NitroMed Heart Failure Advisory Board; Leadership Council for Improving Cardiovascular Care (LCIC) Executive Committee, Schering-Plough; GlaxoSmithKline; AstraZeneca $A B$; Abbott

Speakers Bureau (CME): Pfizer, Novartis, Merck, Bristol-Myers Squibb, Eli Lilly, NitroMed, CV Therapeutics

\section{REFERENCES}

1 Nkomo VT, Gardin JM, Skelton TN, et al. Burden of valvular heart diseases: population-based study. Lancet 2006;368:1005-11.

2 American Heart Association. Heart disease and stroke statistics - 2006 update. Dallas, Texas: American Heart Association, 2006.

3 Williams MA, Fleg JL, Ades PA, et al. Secondary prevention of coronary heart disease in the elderly (with emphasis on patients $\geqslant 75$ years of age). An American Heart Association Scientific Statement from the Council on Clinical Cardiology Subcommittee on Exercise, Cardiac Rehabilitation, and Prevention. Circulation 2002;105:1735-43.

4 Wenger NK. Enrollment and maintenance of elderly patients in cardiovascular clinical trials. Am J Geriat Cardiol 2006;15:352-6.

5 Foody JM, Roc MT, Chen AY, for the CRUSADE Investigators, et al. Lipid management in patients with unstable angina pectoris and non-ST-segment elevation acute myocardial infarction (from CRUSADE). Am J Cardiol 2005;95:483-5. 